\title{
TEC fluctuation during magnetically disturbed events of April 2010 on high latitude station.
}

\author{
Prakash Khatarkar ${ }^{1 *}$, Varsha Kachneria $^{1}$, Harleen Kaur ${ }^{1}$, Roshni Atulkar ${ }^{2}$, \\ P.K. Purohit ${ }^{2}$ and A.K. Gwal ${ }^{1}$ \\ ${ }^{I}$ Space Science Laboratory, Barkatullah University, Bhopal (MP), India \\ ${ }^{2}$ National Institute of Technical Teachers' Training and Research, Bhopal (MP), India
}

\begin{abstract}
The ionospheric parameters, strongly dependent on the solar conditions and on the complex behavior of the magnetosphere, are very interesting for studying the complex phenomena and high degree of space time variability of the ionosphere occurring at the high latitude regions. In order to see the behavior of the high latitude ionosphere during the geomagnetically active events of extreme minimum phase of solar cycle 23 and starting phase of $24^{\text {th }}$ solar cycle, in April 2010 moderate geomagnetic storms have been identified and represented in fairly well detail in the present investigation. The ionospheric observations have been obtained from dual frequency GPS, installed and operated at Indian scientific base station Maitri $\left(71.45^{\circ} \mathrm{S}\right.$ and $\left.11.45^{\circ} \mathrm{E}\right)$ Antarctica, during December. 2009 to December 2010. Situated on auroral region mainly two parameters from the GPS observations, (I) Total Electron Content (TEC), (II) Total Electron Content Rate (TECR) have been considered for the present study. On storm days, the percentage deviations of TEC from geomagnetically quiet days at high latitudes shows the variations of ionosphere in terms of positive and negative ionospheric storms. The results therefore showed the dependence of occurrence characteristics of TEC on the solar activity.
\end{abstract}

Keywords: Geomagnetic Storm, TEC, High latitude ionosphere

\section{Introduction}

The terrestrial ionosphere may be divided broadly into three regions that have rather different properties (Hunsucker and Hargreaves, 2003): the equatorial zone, within $20^{\circ}$ of the magnetic equator, the highlatitude zone, pole-ward of about $60^{\circ}$ geomagnetic and the temperate zone in between. The high latitude zone may be sub-divided into the auroral zone (approximately $60^{\circ}-70^{\circ}$ magnetic) and the polar cap (Pole-ward of the auroral zone). Maitri station is an auroral station from the geographic and geomagnetic point of view. As a result, physical conditions very useful for understanding more clearly the behavior of the Antarctic ionosphere. The $\mathrm{F}$ region ionosphere is the most important region for trans-ionospheric radio propagation, since plasma density is the highest in this region. The exceptionally low solar activity provides the unique opportunity to observe the response of auroral and high latitude ionosphere in the Antarctica sector to the moderate geomagnetic storms. This may help to understand the physical mechanisms operating under the influence of low geomagnetic activity over the region considering the negligible effects from other potential input sources. Our task is to find the link between space weather parameters and the characteristics of ionospheric irregularities. The ionospheric behavior at high latitude is dependent on the factors under the control of geomagnetic conditions, like polar cap convection, and those dependent on geographic location, like production and neutral wind.

Sun is the major source that controls the near earth space and drives its weather and climate. Coronal mass ejections (CMEs) from the Sun interact with the solar wind and the interplanetary magnetic field (IMF) during their propagation; disrupt the flow of the solar wind. Fast CMEs (faster than approximate $500 \mathrm{~km} / \mathrm{s}$ ) eventually drive shocks on the Earth's magnetosphere and trigger geomagnetic storm. Earth's magnetosphere, thermosphere and ionosphere are driven mostly by the energy source of the Sun. In general, moderate and intense Geomagnetic storms are mainly related with the passing interplanetary coronal mass ejections (ICMEs) driving shock waves (Russell et al., 1974; Echer and Gonzalez, 2004; Echer et al., 2008). Based on in situ observations identify three sections in the large scale structure of a shock ICME event associated with the origin of a Geomagnetic storms: (1) the interplanetary (IP) shock wave, (2) the sheath region behind the shock, and (3) the main body of the ICME. During geomagnetic disturbances, such as geomagnetic storms and sub-storms, the energy inputs from the magnetosphere can have dramatic effects to the upper atmospheric environment. One of such effects is the changes in ionospheric electron density that can perturb communication and navigation systems. Large geomagnetic storm, among other things, cause electrical power outages and damage communication satellites. Since every geomagnetic storm has unique character and therefore, forecasting the response of ionospheric parameters like total electron content (TEC) and scintillation (S4) are the major issues 
in space weather studies. The response of the ionosphere to magnetic storms is important factor for understanding the energy coupling process between the Sun and the Earth to forecasting space weather changes.

Ionospheric storm is a global scale ionospheric disturbance of total electron content (TEC), electron peak density and peak height during geomagnetic storm, resulting from different dynamical and chemical processes, such as energetic particle precipitation, changes of electric field and current systems, traveling atmospheric disturbances, thermospheric circulation and composition changes, etc. These factors make ionospheric storms complicated. Ionospheric storms stem from various space weather phenomena that include Coronal Mass Ejections, coronal holes and the background solar wind speed; they are usually (but not always) related to the 11 year solar cycle. In December 2008 solar cycle 24 started. And it will reach its maximum in 2013.

\section{Data Observation And Methodology:}

The TEC is computed from the combined L1 (1575.42 MHz) and L2 (1227.60 MHz) pseudo ranges and carrier phase. This slant total electron content (STEC) was then converted to equivalent vertical total electron content (VTEC) given by:-

$$
\mathrm{VTEC}=\cos \chi \times \mathrm{STEC}
$$

The ionospheric variability of the ionosphere can be estimated by different way, in present study consider $40^{\circ}$ for VTEC for obtaining better result.

The disturb degree was estimated by the percentage deviation from the 5 Quiet days average of the same month with observed TEC of 3-9 April. Where $\mathrm{TEC}_{\text {quiet }}$ is hourly average of five quietest days of that particular month and $\mathrm{TEC}_{\text {disturbed }}$ is hourly average of TEC of magnetic events. We used in this analysis the Rate of Total Electron Content (TECR) based on standard deviation of ROT, (Pi et al., 1997).

The southward magnetic field IMF Bz ensures the transportation of solar wind energy in to earth's magnetosphere, which is the primary cause of the formation of geomagnetic storm.

\section{Results And Discussion:}

\section{Effect of geomagnetic storm on VTEC}

We have analyzed GPS data recorded at Maitri Antarctica throughout the year from December 2009 to December 2010 (starting of 24 th solar activity). We had taken monthly five quiet and five disturbed day total $65+65=130$ day and TEC calculated hourly average basis. Figure ( 2 and 3 ) shows quiet and disturbed day TEC is more fluctuation than quiet days. The purpose of this work to examine the TEC variation depends on local time, latitude and local magnetically disturbed conditions. we present the details of moderate geomagnetic storm and their effects which occurred on 5 April 2010 (with a minimum Dst -73 nT, AE-1347.

The storm events were considered in three main phases, namely, the initial phase, the main phase and the recovery phases. Although it is sometimes difficult to identify the beginning of the recovery phase, therefore we shall consider the main phase and the recovery phase together. In the first week of April 2010, one of the most geomagnetic storm of the solar cycle 24 has occurred. The storm starts on April 5, 2010 with SSC at 0700, followed by a main phase at 08:00 UT. There are successive sub magnetospheric storm denoting an intense auroral ionosphere activity indicated by observed value of $\mathrm{AE}=\mathrm{AU}+\mathrm{AL}$ indexes.

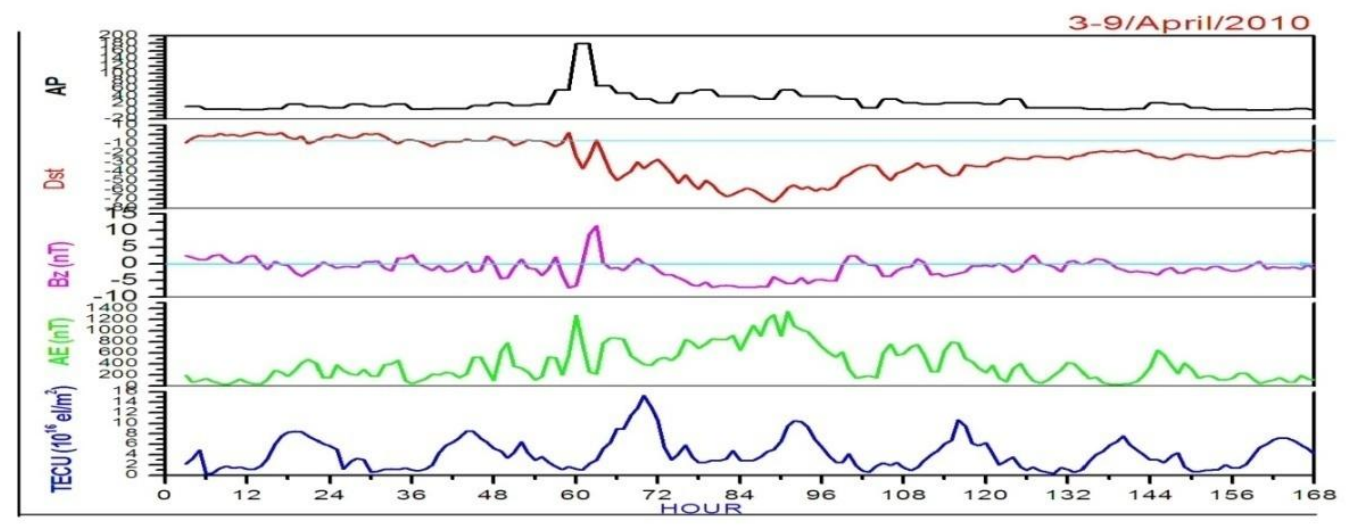

Figure 1: Geomagnetic Storm of 5 April, 2010- VTEC, AE, Bz, Dst, Ap

In this results are presenting some minor geomagnetic disturbances during starting of $24^{\text {th }}$ year solar year 2010. The effects are clearly visible on ionospheric total electron content with geomagnetic indexes during the magnetic storm period. When the solar energetic particles entered in the Earth magnetic field line and 
produced an electric current in the polar region. This current produces disturbances in the magnetosphere and ionosphere. During these disturbances the TEC shows the enhancement. The time period of these storms are few hours to few days. Cause of this short magnetic disturbance, the solar wind was very low down and velocity of energetic particle is less compare to strong magnetic storm. In other words magnetic disturbance was not as much, compare to high solar activity and magnetic field index shows short time fluctuation in the high latitude region during the solar minimum periods. This magnetic variation also affects to polar ionosphere with in miniature force and TEC suddenly increases due to storm period and drop down in its regular pattern after magnetic storm.

The effect of the geomagnetic activity on TEC is not always positive. The inhibition and enhancement of the irregularities during geomagnetic disturbances can be attributed to change in the current system (Aarons, 1991) several scientist found similar result about the relation between TEC and geomagnetic activity. During the geomagnetic disturbances the total electron content are suppressed in pre-midnight and increase in postmidnight (Gwal et al., 2010 and 2004, Aurons et al., 1980; Rastogi et al., 1981, Kumar et al., 1993).

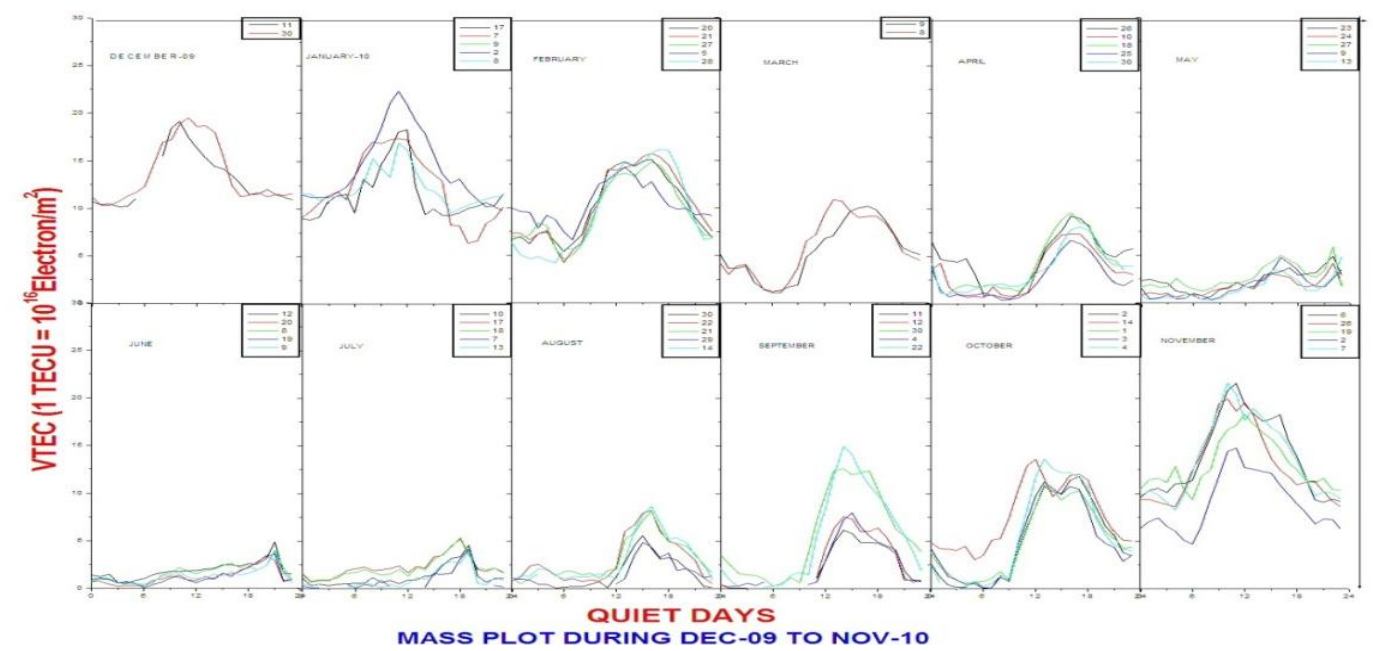

Figure - 2: TEC mass plot of most quiets days during December-2009 to November-2010

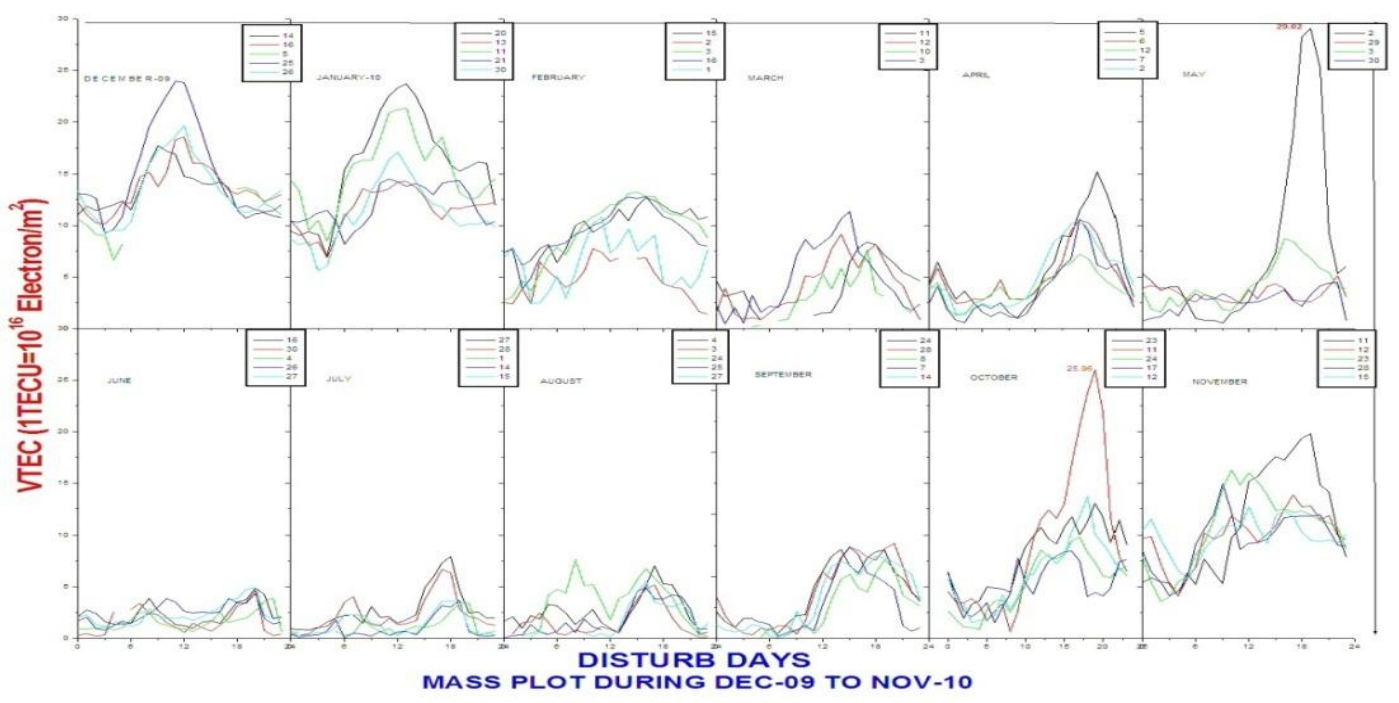

Figure - 3: TEC mass plot of disturb days during Dec- 2009 to Nov- 2010 


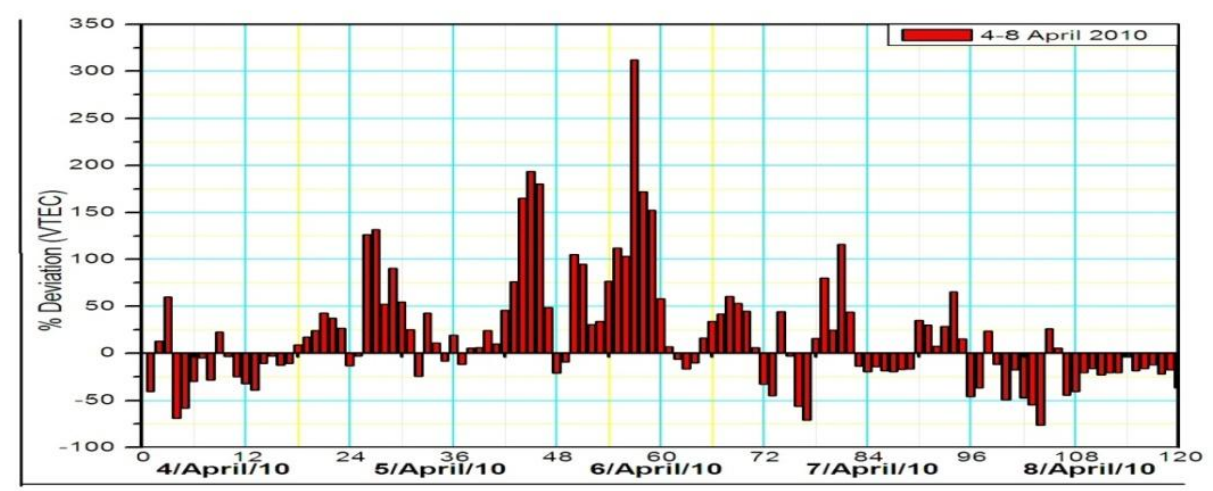

Figure - 4: Hourly percentage deviation of VTEC during Magnetic storm of April 3-9, 2010

The 5 April 2010 magnetic storm is considered as moderate geomagnetic storm occurred between (7:00 to U10:00 UT). The north south component Bz of the interplanetary magnetic field (IMF) exhibited incursion to the south that remain about 4 hour reaching a minimum value -7.2 at 8:00 UT. It turns to northward for 2 hour, reaches up to $11.4 \mathrm{nT}$, and suddenly turned southward. AE and Dst value suggest that 5- 6 April both the days are disturbed day. Magnetic field and $\mathrm{Kp}(77 \mathrm{nT})$ indicate the geomagnetic storm is moderate in figure 1 the Auroral Electrojet (AE) index reached its maximum value 1278 nT at about 9:00 UT on 5 April and second peak of AE index has been observed $1092 \mathrm{nT}$ on 6 April 2010 and maximum value of AE reached on this time 1347 nT. The strong variation in the TEC was caused by the presence of large scale ionospheric structure of enhanced electron density in the polar ionosphere. These structures were associated with the occurrence of polar patches. The rate of polar patches is more when IMF Bz is southward or (Kp > 4) Rodger et al., (1994). Priderson et al., (2000), and Prikryl et al., (2005)., observed that the patches were travel through the ionospheric polar caps under the influence of the high latitude convection. These patches are typically considered to be of the order of $100-1000 \mathrm{kms}$ in horizontal extent. The traveling speed of the patches is between $300-900 \mathrm{~ms}^{-}{ }^{1}$ these polar patches are responsible for scintillation. We found -2 to 2 TECU/min between 01:00 to 02:00 UT and continually Fluctuation until 6 April with range of -1.5 to $1.5 \mathrm{TECU} / \mathrm{min}$. In the Fig. 4 hourly percentage VTEC deviation plotted, the hourly average value of Vertical Total electron content maximum for the period $(\sim 15$ TECU, 1 TECU $=10^{16}$ eletron $/ \mathrm{m}^{2}$ ) of magnetic storm on 5 April and percentage deviation varying $\sim 176$ to $325 \%$ and on the recovery phase of the storm VTEC show the positive deviation on 6 April 2010. Figure ( 1) illustrated the maximum value during the geomagnetic storm reached its highest value at about 24 TECU on 5 April 2010.Entire plasma parameter indices figure (4) show Hourly percentage deviation of VTEC of April 3-9, 2010 increases and fluctuation during magnetic storm.

\section{Conclusion}

Our analysis of TEC fluctuation has shown, strong phase fluctuation observed when Ap, Kp, AE and all geomagnetic and solar indices reached its maximum value and intensity of TEC fluctuation strongly increase relative to quite condition due to presence of medium and large scale irregularities in the ionosphere. As a measure of TEC fluctuation, the vertical TEC during the magnetic storm clearly indicated Figure (2and 3) increased values and TEC mass plot of most quiets days and disturbed days during December-2009 to November-2010 and pick shifted toward day to night side.

\section{Acknowledgment:}

The author (Prakash Khatarkar) is highly thankful to National Physical Laboratory (NPL), New Delhi and National Centre for Antarctic and Oceanic Research (NCAOR), Goa for providing opportunity to be a member of $29^{\mathrm{Th}}$ Indian Scientific Expedition to Maitri Antarctica. The financial assistance received from University Grants commission, New Delhi, India, through Rajiv Gandhi National Fellowship (SRF) program is also acknowledged

\section{References}

[1]. Aarons, J., Mullen J.P., Koster, J.R., (1980) "seasonal and geomagnetic control of equator scintillation in to longitude sectors. J. Atmos. Terr. Physics. 42, 861-866.

[2]. Adeniyi JO.,(1986), Magnetic storm effects on the morphology of the equatorial F2 - layer. Journal of atmospheric and terrestrial physics; 48(8):69-702).

[3]. Aurons. J. (1997), Global positioning system phase fluctuations at auroral latitudes. J.Geophys. Res 102. A8, PP.-17.219-17.231. 
[4]. Echer, E., and W. D. Gonzalez (2004), Geoeffectiveness of interplanetary shocks, magnetic clouds, sector boundary crossings and their combined occurrence, Geophys. Res. Lett., 31, L09808, doi:10.1029/ 2003GL019199.

[5]. Echer, E., W. D. Gonzalez, B. T. Tsurutani, and A. L. C. Gonzalez (2008), Interplanetary conditions causing intense geomagnetic storms (Dst $=-100 \mathrm{nT}$ ) during solar cycle 23 (1996-2006), J. Geophys. Res., 113, A05221, doi:10.1029/2007JA012744.

[6]. Gwal A.K. Amit Jain, (2010), GPS scintillation Studies in the Arctic region during the first winter phase 2008 Indian Arctic Expedition. Polar science XX, 1-14.

[7]. Gwal A.K., Smita Dubey , Rashmi Wahi, (2004) A study of L-band scintillations at equatorial latitudes. Advances in Space Research. 34, 2029-2095.

[8]. Hunsucker, R.D. and J.K.Hargreaves, J.K., (2003), The High-Latitude Ionosphere and its Effects on Radiopropagation, Cambridge.

[9]. Kumar S., Singh A.K., Pawan Chouhan (1993) Multi-station Analysis of VHF radio wave scintillation at low latitude. J. of radio space Phys. 22, 267-272,

[10]. Pi, X., A.J. Mannucci, U.J. Lindqwister and C.M. Ho, (1997), Monitoring of global ionospheric irregularities using the worldwide GPS network, Geophys. Res. Lett. 24, 18, 2283-2286.

[11]. Prikryl, P., Mac Dougall J. W., Grand I.F., Steele D.P., Sofko G.J. and Greenwald R.A., (1999), Observation of Polar patches generated by solar wind Alfaven wave coupling to the dayside magnetosphere. Annals Geophys., 17,463-489.

[12]. Rastogi. (2006), Magnetic storm effects at equatorial electro jet stations, Earth planets space;58:645- 57.

[13]. Rodger. A.S. Pinnock M., Dudeney J.R., Baker K.B., Greenwald A.R.,( 1994). A new mechanism of polar patch formation. J. of Geophys.Res., 99, 2335-2336.

[14]. Russell, C. T., R. L. McPherron, and R. K. Burton (1974), On the cause of geomagnetic storms, J. Geophys. Res., $79(7), 1105$ 1109. 